\title{
Pathophysiological mechanism in anorexia nervosa identified by neuropathological analysis
}

\author{
I. Kawakami ${ }^{1,2,3}$, K. Umeda ${ }^{3}$, Y. Murahashi ${ }^{3}$, K. Niizato ${ }^{3}$, K. Oshima ${ }^{3}$, M. Hasegawa², S. Murayama ${ }^{1}$, S. Iritani $^{4}$ \\ ${ }^{1}$ Tokyo Metropolitan Institute of Gerontology, Dept. of Neuropathology, Tokyo, Japan. \\ ${ }^{2}$ Tokyo Metropolitan Institute of Medical Science, Dementia Project, Tokyo, Japan. \\ ${ }^{3}$ Tokyo Metropolitan Matsuzawa Hospital, Dept. of Psychiatry, Tokyo, Japan. \\ ${ }^{4}$ Nagoya University Graduate School of Medicine, Dept. of Psychiatry, Nagoya, Japan.
}

\section{Background}

Anorexia nervosa (AN) is a serious eating disorder characterized by self-starvation and extreme weight loss, and has the highest mortality ratio among all psychiatric disorders. Recent functional/structural neuroimaging research indicates that impaired cognitive-behavioral flexibility is associated with intact functioning of cortico-striatal loop systems, including the caudate, pallidum, nucleus accumbens, prefrontal area and anterior/posterior cingulate. However, the pathophysiology involves interaction of multiple developmental, genetic, environmental, and neurobiological factors, and the details remain unclear. We aimed to clarify the pathophysiology of AN by investigating the neuropathology of patients' brains.

\section{Materials and Methods}

Brain sections of 3 AN patients were stained with hematoxylin and eosin and Klüver-Barrera stain. Immunohistochemistry was performed with antibodies to tyrosine hydroxylase (TH) and glial fibrillary acidic protein (GFAP) in serially cut paraffin sections. Primary antibody labelling was visualized using $0.2 \%$ 3,3'-diaminobenzidine as the chromogen in combination with an Envision Plus kit. For confocal microscopy, Alexa Fluor secondary antibodies (Thermo Fisher Scientific) were used. Ethical issues were carefully considered in this study.

\begin{tabular}{|l|l|l|l|l|l|}
\hline & $n$ & $\begin{array}{l}\text { Sex } \\
(\text { female/m } \\
\text { ale, } \%)\end{array}$ & $\begin{array}{l}\text { Onset } \\
(y, \text { Mean } \pm \text { SD })\end{array}$ & $\begin{array}{l}\text { Death } \\
(y, \text { Mean } \pm S D)\end{array}$ & $\begin{array}{l}\text { Duration } \\
(y, \text { Mean } \pm S D)\end{array}$ \\
\hline $\begin{array}{l}\text { Anorexia } \\
\text { nervosa }\end{array}$ & 3 & $\begin{array}{l}0 / 3 \\
(100.0)\end{array}$ & $18.7 \pm 4.2$ & $36.3 \pm 11.0$ & \pm \\
\hline Control & 7 & $\begin{array}{l}2 / 5 \\
(40.0)\end{array}$ & - & $58.6 \pm 11.2$ & - \\
\hline
\end{tabular}

\section{Results}

Macroscopically, AN patients show slight frontal atrophy with enlargement of lateral ventricles. Average brain weight was $1183 \pm 63.5 \mathrm{~g}$.

Histologically, two main neuropathological changes were seen:

\section{Pathological changes in dopaminergic pathways}

In AN patients' brains, neuronal loss and gliosis were seen in the frontal cortex, anterior cingulate, and caudate nucleus. Especially, the nucleus accumbens showed moderate/severe changes, compared to controls. Adjacent sections stained for TH and GFAP indicate that gliosis preferentially occurs in areas where the fine, mesh-like TH staining is relatively light.

\section{Mild stage of Wernicke's encephalopathy (WE)}

WE is a neuropathological disease caused by thiamine deficiency. AN patients showed gliosis/hemorrhages in the walls of the third ventricle, the mammillary bodies, and the floor of the fourth ventricle. One case presented central pontine myelinolysis.

Senile changes, including neurofibrillary tangles/senile plaques, TDP and $\alpha$-synuclein pathology, were mild in all cases.
Pathological changes in dopaminergic pathways

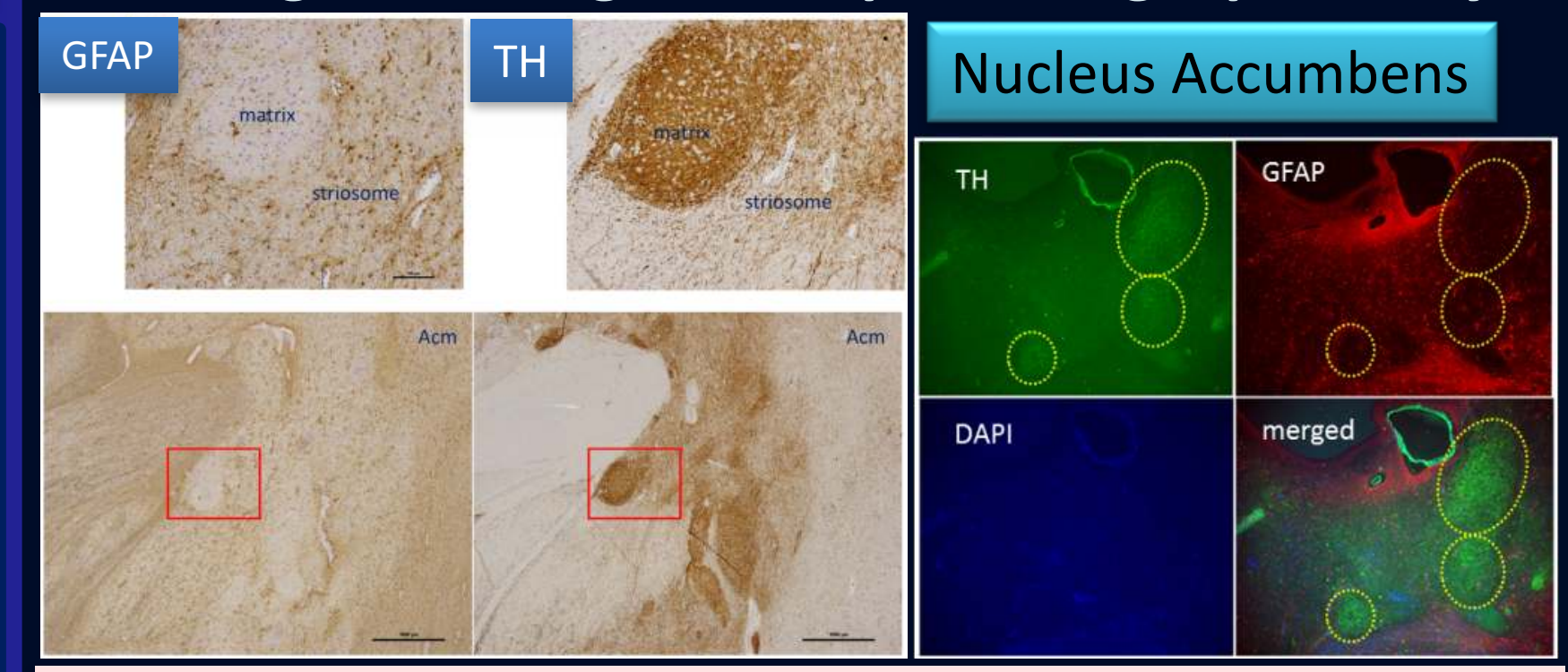

Projections

TH-: Striosome ( $\leftarrow$ Hippocampus) $\mathrm{TH}+$ : Matrix ( $\leftarrow$ prefrontal cortex) Gliosis shows up in meso-limbic pathways

Regions associated with dopaminergic pathways

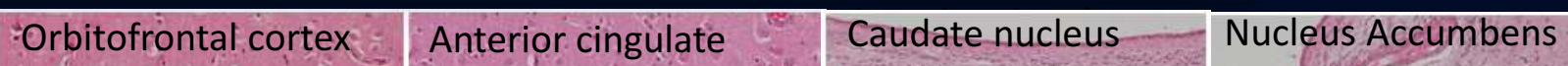

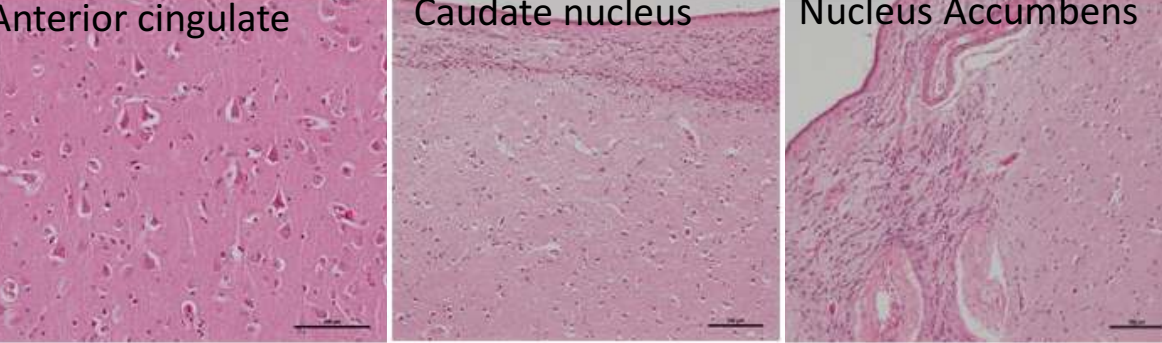

Ischemic neurons and mild/moderate gliosis in regio

Mild stage of Wernicke's encephalopathy
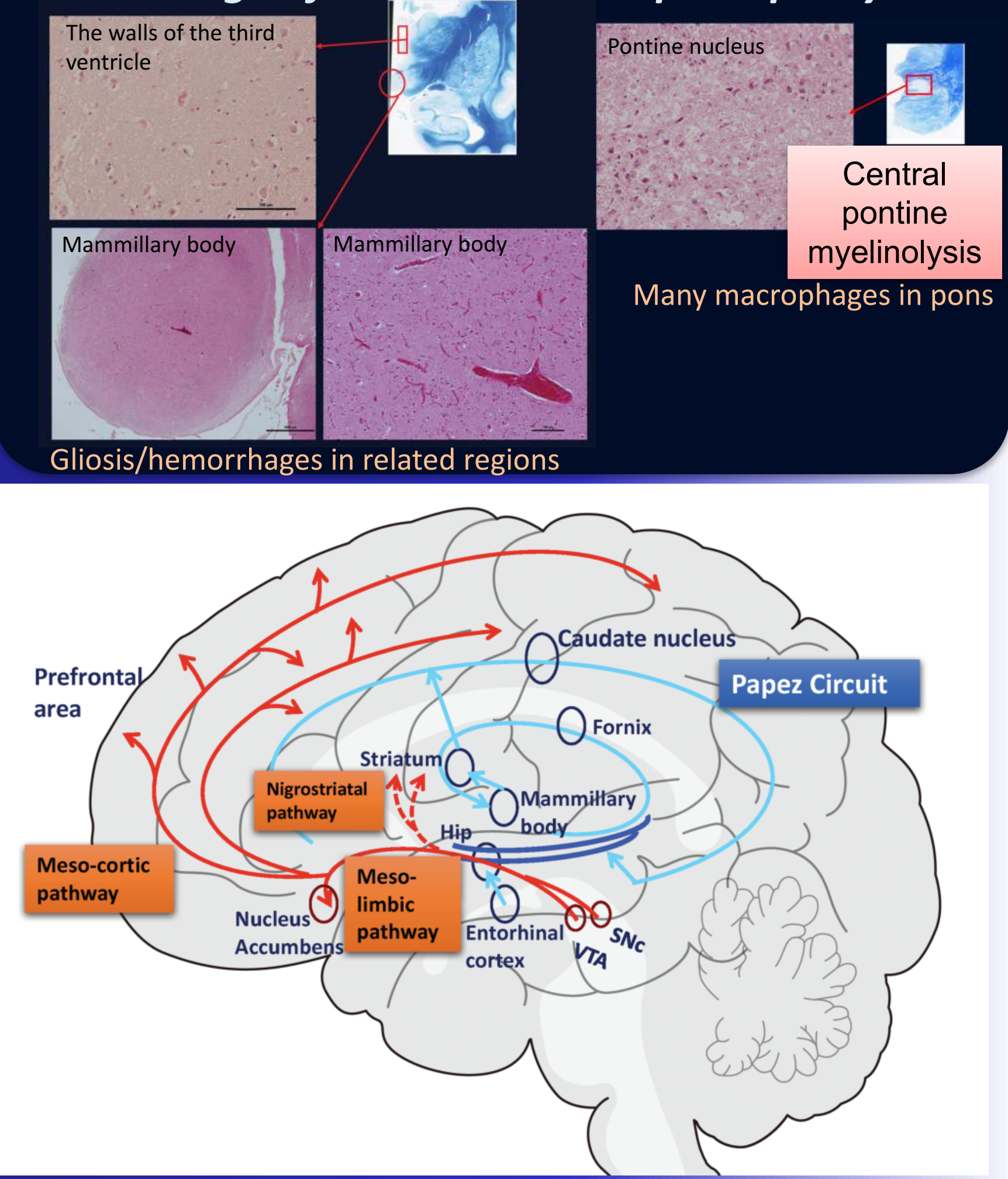

\section{Conclusion}

Our results indicate that impaired functional connectivity in a reward-related network may cause the neuropsychiatric symptoms in AN patients. Furthermore, the affected areas in WE correspond with the regions related to the Papez circuit, a closed neural circuitry that controls emotions and memory retention. These findings should be helpful in developing more effective treatments for AN. 